\title{
Caracterización del sistema de producción caprina y lineamientos de una propuesta de desarrollo en el valle de Cañete
}

\author{
Description of the goat production system and guidelines \\ for a development proposal in the Cañete valley
}

\author{
José Sarria'; Germán Navia²
}

\begin{abstract}
Resumen
El objetivo del presente trabajo fue caracterizar el sistema de producción caprina y la elaboración de lineamientos para el desarrollo de dicha actividad económica en el valle de Cañete, en la costa central del departamento de Lima, Perú. La metodología empleada incluyó selección de la muestra, elaboración, aplicación de una encuesta y procesamiento de datos. Se entrevistó a 60 productores y se pudo caracterizar la explotación caprina rastrojera de la zona por medio de análisis estadísticos descriptivos. Se concluye que la crianza es básicamente empírica, con productores de bajo nivel educativo, cuyos rebaños asociados a vacunos y ovinos están conformados, en promedio, por 46,85 cabezas. La comercialización, aspecto crítico, es realizada a través de intermediarios. Con el método de análisis de agrupamientos y una selección de variables relevantes (edad, estudios, área, rebaño, vacunos, ovinos, corrales, leche, ordeño, tiempo de producción) se llegó a identificar tres grupos de criadores (a) Productores caprinos de mayor escala productiva, (b) Productores caprinos con mayores áreas de terreno y (c) Productores caprinos de mayor edad. Finalmente, se sugiere la urgente necesidad de capacitación técnica, de motivación progresiva del asociacionismo y mejora genética, para facilitar el logro de estos fines prioritariamente.
\end{abstract}

Palabras clave: caprinos; caracterización; desarrollo pecuario; sistemas de crianza.

\begin{abstract}
The purpose of this study was to describe the goat production system and to elaborate guidelines for the development of this economic activity in the Cañete valley, located in the central coast of the Lima department, Perú. The methods used in this study included sampling, preparation, a survey application and data processing. 60 goat producers were interviewed, and the goat production systems was characterized using descriptive statistical analysis. We found that the goat production system in the study zone was empirical, where producers have a low educational level, their herds have mixed cattle and sheep, with an average size of 46,85 animals. The commercialization, critical aspect, was carried out through many intermediate vendors. By using cluster analysis on survey data with selected variables (age, studies, area, herd, bovine, sheep, pens, milk, milking method, and production time) three main producer groups were identified: (a) high scale goat producers, (b) goat producers with larger land areas and (c) older goat producers. Finally, it was suggested to strength technical training, to promote business association, and to implement a genetic improvement program.
\end{abstract}

Keywords: goat; characterization; livestock development; production systems.

\section{Introducción}

En el Perú, la crianza caprina es una de las producciones de menor beneficio económico para sus propietarios. Los criadores de esta especie se encuentran ubicados principalmente en la costa y sierra del país, con explotaciones de baja producción y productividad, debido -entre otros aspectos- a la mala calidad genética, forma de manejo productivo-reproductivo y, en general, a la falta de visión empresarial.

En la zona central de la costa del Perú, se practica la crianza en base al uso de rastrojos de la agricultura; el tamaño de estas explotaciones es de tendencia reducida, con exiguas producciones de leche, generando carne como producto de baja valoración. La problemática de estos productores caprinos es compleja ya que a la precaria tenencia de la tierra, en la mayoría de casos, que ha conducido a una degradación acentuada del suelo (sobrepastoreo con erosión), se suman la carencia de conocimientos tecnológicos para mejorar la producción y la evidente falta de capacidad empresarial (De Gea, 2000).

La poca información general disponible, aunada a la ausencia de conocimientos y a la escasa iniciativa por parte de los ganaderos en cuanto a lograr una adecuada gestión técnico-económica de sus explotaciones, se expresa de muchas formas. Así, los criadores raramente registran datos de funcionamiento (gastos e ingresos, época de cubriciones, alimentación, etc.); mucho menos

\footnotetext{
1 Profesor Principal. Facultad de Zootecnia. Universidad Nacional Agraria La Molina, Lima, Perú. Email: jsb@lamolina.edu.pe

2 Tesista post grado, Producción Animal. Universidad Nacional Agraria La Molina, Lima, Perú. Email: mancocapac02@yahoo.com.ar
} 
controlan individualmente a las cabras. Todo ello influye negativamente en la marcha de la explotación y, por supuesto, dificulta en gran medida cualquier propuesta que trate de aportar conocimientos y mejores prácticas al referido sistema productivo (Mena et al., 2005).

El sector agropecuario en el Perú es el principal generador de empleo, sin embargo se mantiene en crisis permanente; la situación se complejiza por la profundización de los tradicionales problemas de baja producción $\mathrm{y}$ productividad, subempleo y desempleo de los recursos humanos, así como los consecuentes bajos niveles de ingreso de amplios grupos de la población agrícola rural en el país. De allí la importancia, en primer lugar, de buscar caminos lógicos y coherentes con el desarrollo agrícola y pecuario y, en segundo lugar, consolidar el desarrollo rural, íntimamente ligado al primero (Ordoñez, 2002).

El objetivo general de este trabajo es caracterizar el sistema de producción caprina del valle de Cañete, provincia de Cañete, de la región Lima, Perú. Este objetivo amplio se complementa con la identificación de los factores socioeconómicos y productivos que caracterizan y limitan el sistema de producción caprina del valle de Cañete así como detectar sus potencialidades; proponer alternativas de desarrollo para el sistema rastrojero de producción caprina del área en referencia, como modelo replicable en valles y zonas similares, en la intención de mejorar la situación de los criadores caprinos abocados a dicho sistema.

\section{Materiales y métodos Localización}

El área de estudio se encuentra ubicado a 150 kilómetros al sur de la ciudad de Lima, en la provincia de Cañete, entre las coordenadas geográficas de $13^{\circ} 02^{\prime} 21^{\prime \prime}$ latitud Sur y $76^{\circ} 29^{\prime} 21^{\prime}$ ' longitud Oeste. Se distinguen en esta zona, diferentes fisiografías como quebradas, colinas, cerros, bahías y playas. Para efectos específicos del trabajo, se consideraron los distritos de San Luís, San Vicente, Nueva Imperial, Imperial, Quilmaná y Cerro Azul, pertenecientes al área conocida como desierto subtropical (D-ST).

\section{Materiales}

Los principales materiales que se usaron, durante la investigación, fueron los siguientes: Formato de encuesta, computadora, programa SPSS-13, cámara fotográfica, mapas, reportes climáticos y vehículos motorizados.

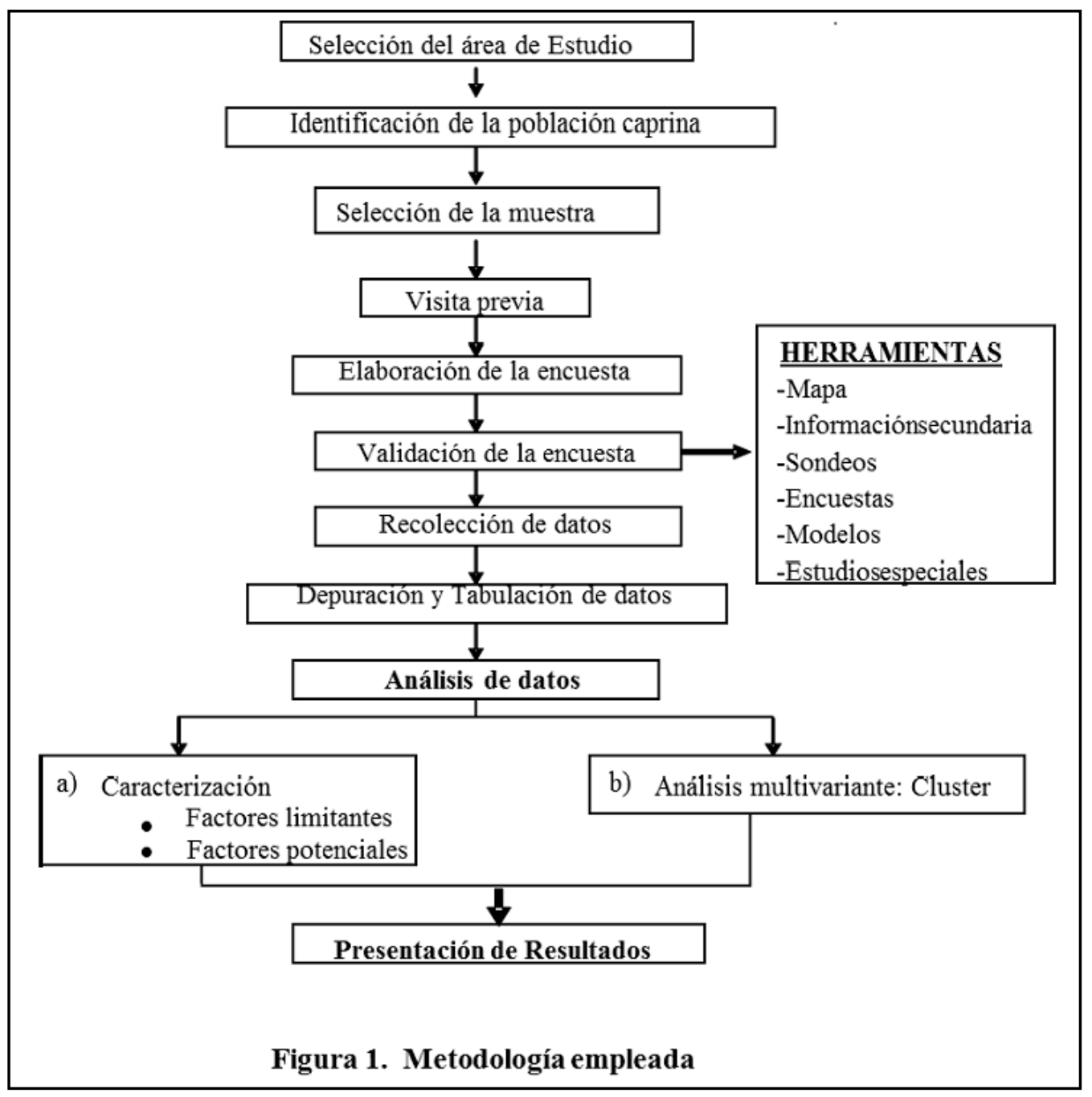




\section{Metodología}

La metodología empleada en este trabajo de investigación incluyó varios pasos secuenciales que van desde la selección del área hasta la caracterización del sistema productivo propiamente (Fig. 1).

\section{Caracterización}

La caracterización es una metodología que permite clasificar la función que cumple cada componente del sistema productivo. Funcionalmente, permite separar tres partes genéricas de un proceso: la entrada de insumos, la transformación de estos y la salida del producto; dos elementos adicionales son el contexto, o entorno externo donde opera el sistema, y los recursos, que son componentes internos del sistema que se usan en el mismo (Ordoñez, 2002).

En el presente caso, la caracterización se realizó para obtener la mayor información posible del sistema en estudio, a fin de conocer sus potencialidades y limitaciones ambientales, demográficas y productivas, entre otras. La información fue detallada, única manera para entender cómo funciona el sistema en cuestión, para luego hacer el respectivo diagnóstico. La caracterización consistió en la ejecución consecutiva de tres etapas genéricas que fueron: el sondeo, la encuesta y, por último, el análisis estadístico e interpretación de resultados. Se entrevistó a 60 criadores por medio de una encuesta estática (entre agosto y octubre 2008). Se recorrió primero los distritos que se encuentran en el litoral, para después hacer lo propio con los que se encontraban hacia el interior del valle (Fig. 2).

La encuesta contó con preguntas agrupadas en temas centrales tales como: (I) Aspectos generales, (II) Sistema de explotación, (III) Proceso productivo, (IV) Comercialización y (V) Proyección y problemática.

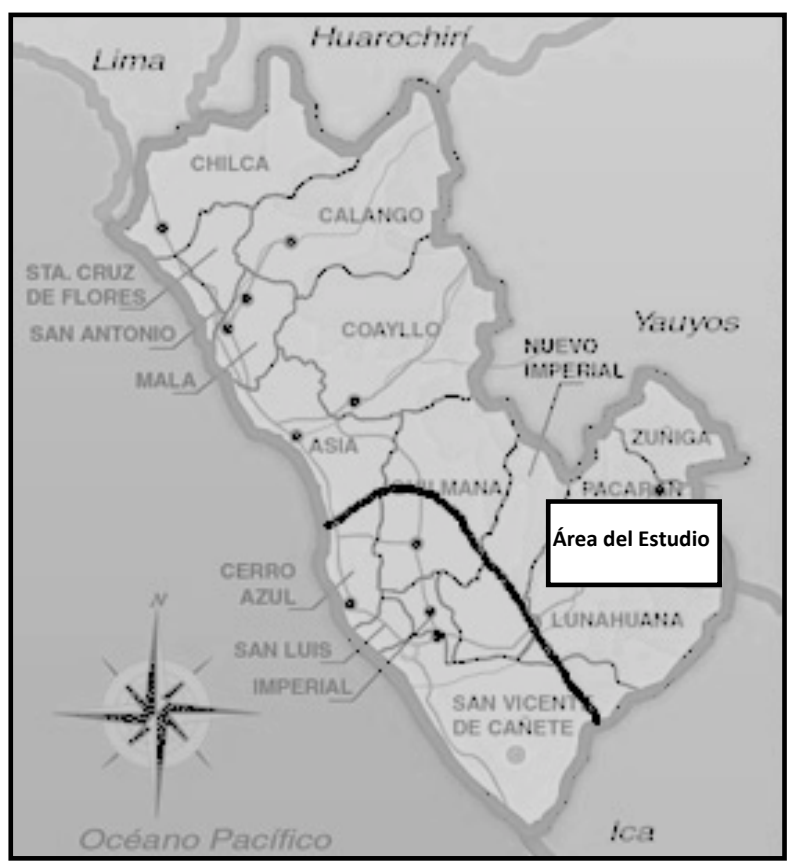

Figura 2. Distritos de Cañete y área del estudio
Para determinar el tamaño de la muestra se utilizó la metodología de muestreo simple aleatorio. Reemplazando los valores establecidos se obtiene:

$$
\mathrm{n}_{0}=\frac{0,5(1-0,5)(1-96)^{2}}{(0,105)^{2}}=87,11=87
$$

Luego se determinó el tamaño de muestra ajustada en función de la población, como sigue:

$$
\mathrm{n}=\frac{87}{1+\frac{87-1}{176}}=58,44=58
$$

En resumen, se obtuvo un tamaño mínimo de muestra de 58 individuos, considerando un nivel de confianza del $95 \%$ y un margen de error de $10,5 \%$; sin embargo, se entrevistaron dos criadores más, con el propósito de cubrir alguna pérdida de datos.

\section{Método de investigación}

Se aplicó el método estadístico descriptivo, con la encuesta como principal herramienta usada. El tipo de investigación empleado fue el no experimental, en el cual no se hacen manejables las variables independientes; es decir, resulta imposible manipular variables. Específicamente, el tipo de diseño no experimental fue la investigación transeccional o transversal (Gómez, 2004).

\section{Tipificación}

La técnica estadística multivariante, también llamada análisis de agrupaciones o análisis de clúster, tiene por objeto formar grupos de individuos homogéneos respecto a una variedad de atributos asumidos conjuntamente (Álvarez y Paz, 1998) con el fin ulterior de estudiar y proponer cambios y mejoras al prototipo de sistemas análogos.

Cabe resaltar que dicho análisis es netamente descriptivo y no inferencial. Con las asunciones previamente descritas, a través de la elaboración del dendograma (representación gráfica que ayuda a interpretar el resultado de un análisis cluster), se procedió a los pasos seguidos en el método interactivo dentro del análisis. Gráficamente, en el eje $\mathrm{Y}$ se encuentran los individuos u objetos en su orden de aparición en el algoritmo, mientas que en el eje X están las distancias, que finalmente permiten identificar, en nuestro caso, los grupos de productores diferenciados.

\section{La propuesta técnica}

Con la información de las encuestas generada y procesada y al conocer las limitaciones y potencialidades de la actividad caprina en la zona, se tuvo una línea de base para la formulación de alternativas y propuestas en el aspecto técnico-productivo y comercial para los establecimientos ganaderos de la especie en estudio, así como para el nivel organizacional de dicho sector. 


\section{Resultados y discusión}

\section{Generalidades del sistema de producción}

Todo sistema es la integración de subsistemas, componentes y factores de ingreso (input) y de salida (output) que están interrelacionados; para el caso de Cañete y la producción caprina, se obtuvo el esquema presentado en la Fig. 3.

Análisis descriptivo de los factores socio-económicos de los criadores

Los productores caprinos del valle de Cañete tienen un promedio de edad de 53,12 años, datos que se acercan a los encontrados en España (García et al., 1999), donde la edad media hallada fue de 50 años. En total, 27 productores $(45,76 \%)$ tienen una edad que oscila entre los 45 y 59 años, mientras que solo 15 productores $(25,42 \%)$ fluctúan entre los 29 y 44 años; el hecho de que los productores del grupo más joven sean solo 15 demostraría que los cabreros jóvenes estarían migrando a las grandes urbes en busca de un mejor porvenir, alternativo al actual. Finalmente, también se tiene un importante $27,12 \%$ de personas de la tercera edad (60 a 74 años) y 1,69 \% mayores de 75 años dedicados a esta actividad (Fig. 4). El género femenino tiene más alta presencia en edades mayores.
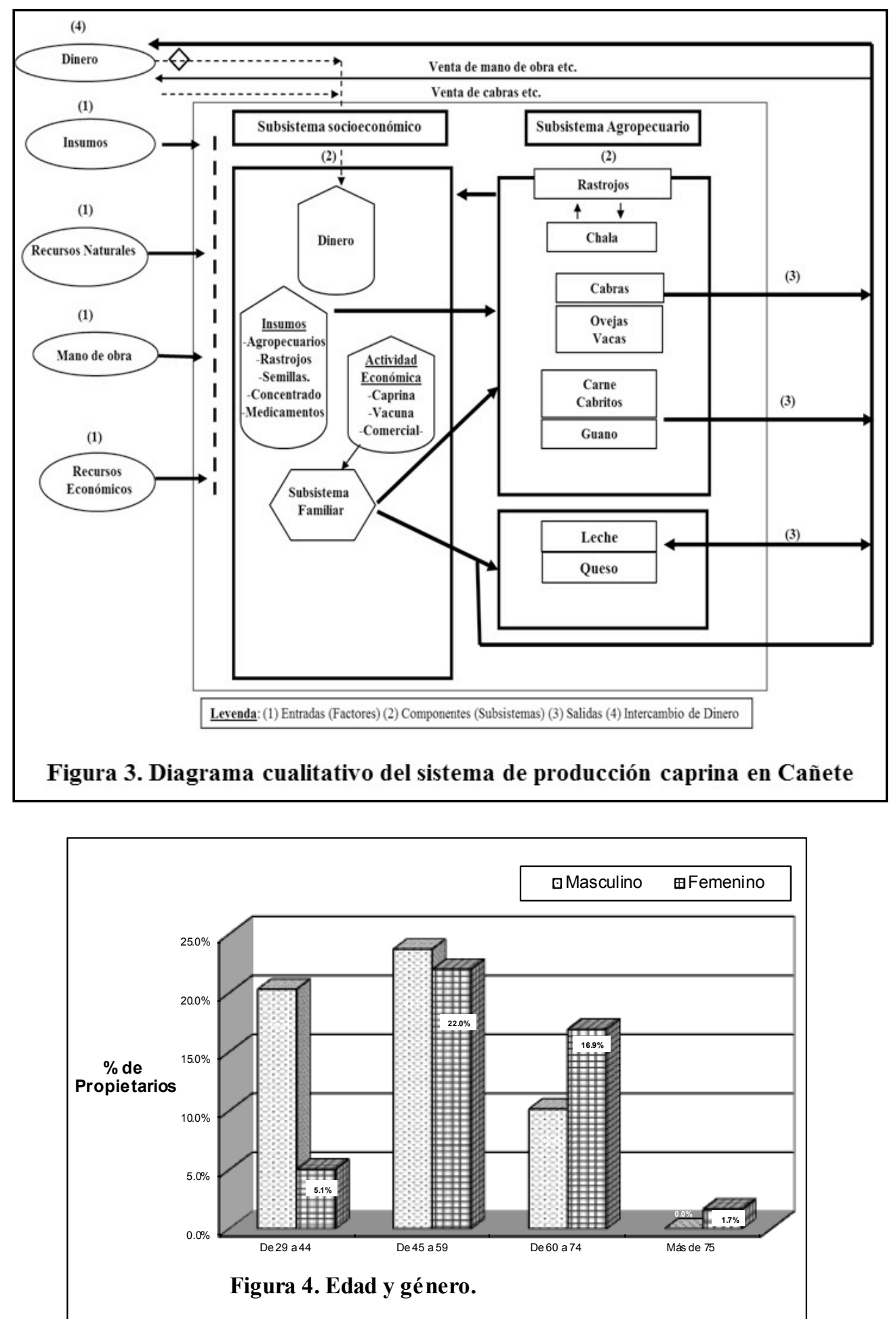
Con respecto a las extensiones de terreno que ocupan los criadores caprinos del valle de Cañete, se puede observar que más de la mitad de los productores tienen un área por debajo de 0,1 ha. Esto se debe a las migraciones que hicieron en años anteriores cuando practicaban la ganadería trashumante para, posteriormente, convertirse en rastrojeros, asentándose en pequeñas parcelas eriazas en la línea del litoral o en los límites de los valles que colindan con la cordillera, quedando como propietarios de estos lotes por posesión. Solo cerca del $7 \%$ de los productores tienen terrenos por encima de 0,5 ha (Fig. 5). Los resultados difieren a los encontrados en el sistema de bosque seco en Piura donde se reportó que solo el $11,76 \%$ poseían entre 0 y 0,99 ha, mientras el resto de criadores tenían extensiones mayores a una hectárea (Venegas, 2002).

En cuanto a aspectos de crédito y asociativismo, los datos indicaron que los productores del valle en estudio no cuentan con ningún programa crediticio, además la gran mayoría de ellos $(89,8 \%)$ no se encuentran asociados a ninguna agremiación de productores caprinos (Tabla 1).

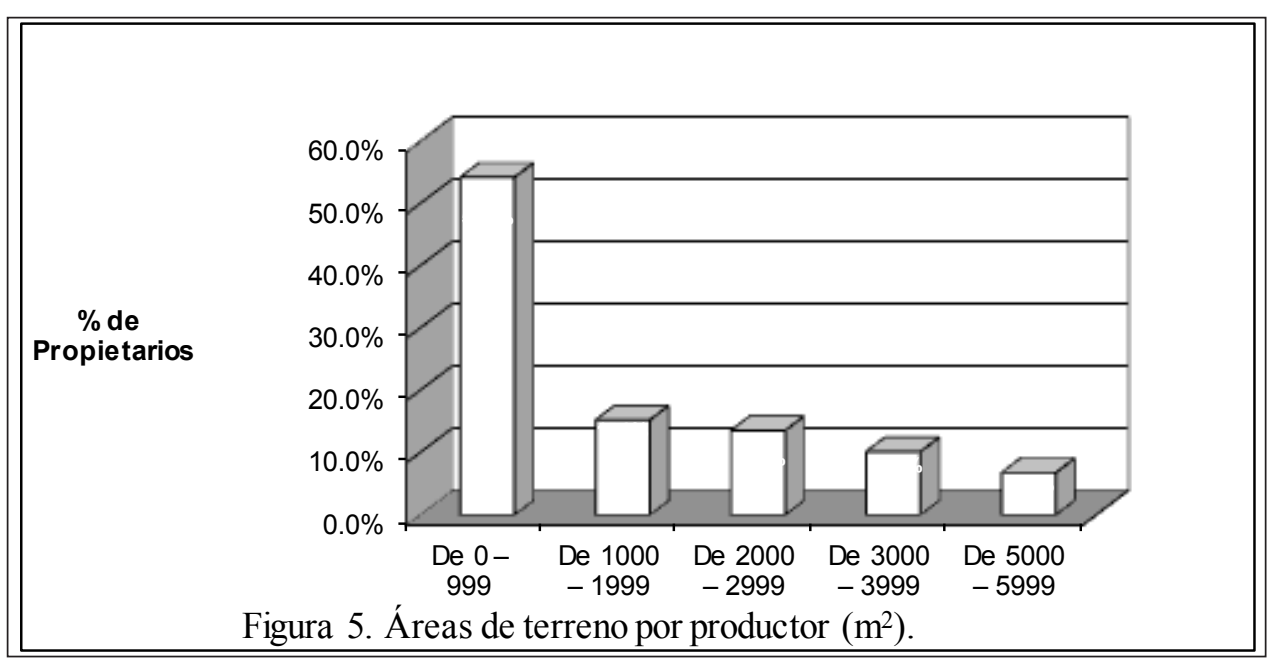

Tabla 1. Asociativismo

\begin{tabular}{|c|c|c|}
\hline $\begin{array}{c}\text { Pertenece a alguna } \\
\text { asociación }\end{array}$ & $\begin{array}{c}\mathbf{N}^{\mathbf{0}} \text { de } \\
\text { Productores }\end{array}$ & $\begin{array}{c}\text { \% de } \\
\text { Productores }\end{array}$ \\
\hline $\mathrm{Si}$ & 6 & $10,2 \%$ \\
\hline $\mathrm{No}$ & 53 & $89,8 \%$ \\
\hline Total & $\mathbf{5 9}$ & $\mathbf{1 0 0} \%$ \\
\hline
\end{tabular}

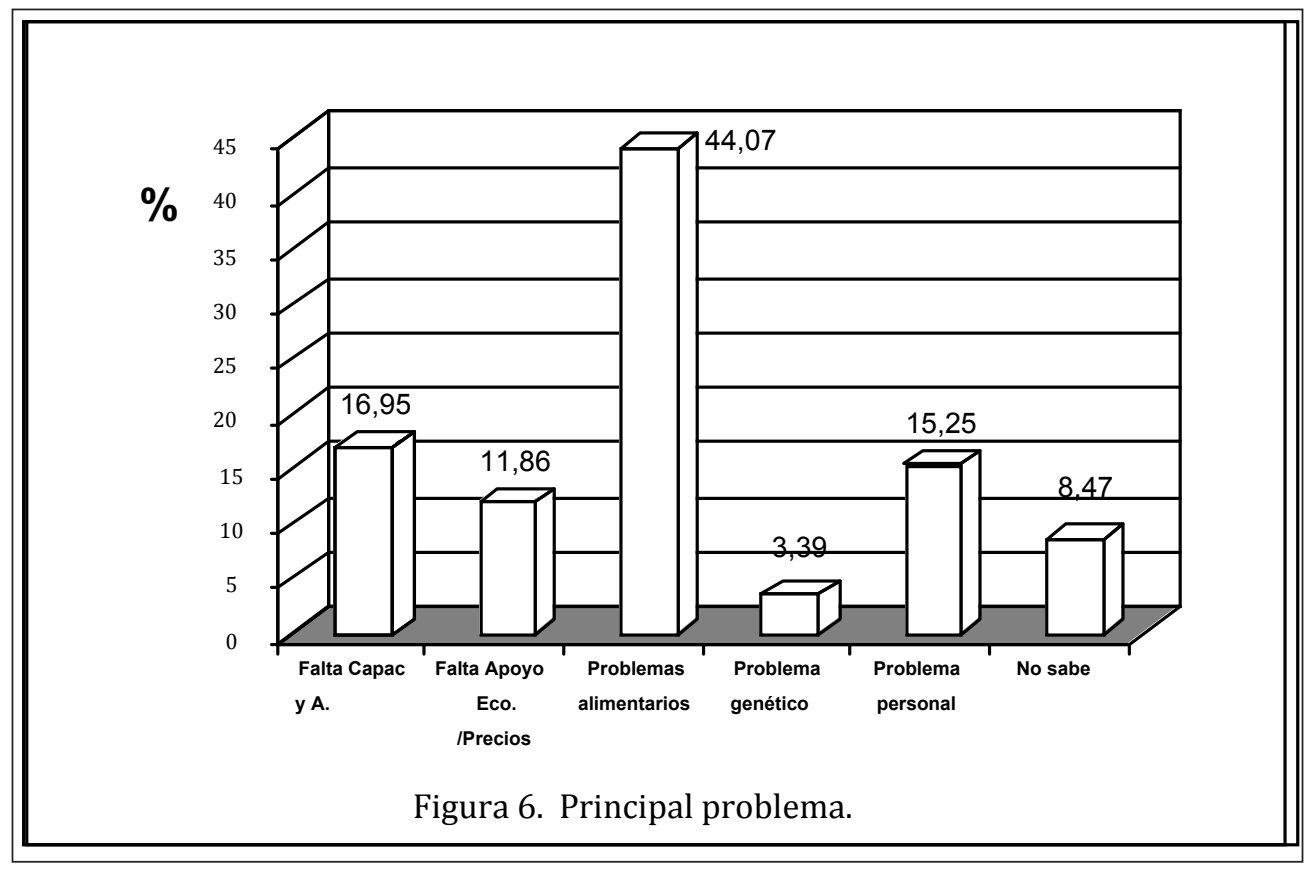


El 44,07 \% de los criadores caprinos del valle de Cañete consideran que el principal problema que afrontan es el alimentario, ya que escasea el rastrojo o forraje una buena parte del año. Solo el 3,39 \% consideran que el problema básico es la falta de buena genética para su rebaño; otras razones son la escasez de capacitación, la ausencia de apoyo económico y los problemas personales, tal como se observa en la Fig. 6.

\section{Análisis descriptivo de los factores productivos del sistema}

En lo concerniente al tipo de crianza (Tabla 2), se encontró que el mayor porcentaje de explotaciones eran rastrojeras $(84,7 \%)$, ya que la costa peruana, en especial la zona central, se caracteriza por tener abundancia de residuos de cosecha agrícola, que son consumidos preferentemente por las cabras. El tipo de crianza intensiva solo registra una mínima porción $(6,8 \%)$; mientras que la explotación trashumante fue del 8,5\%. Este último dato guarda relación con los encontrados en España (García et al., 1999), en donde la trashumancia se realizaba, en 7,97\% de los casos, caminando y en el 11,11 \% usando vehículos de carga (camionetas o camiones). La trashumancia combinada con el rastrojeo se realiza principalmente durante los meses de marzo a junio, época en la cual escasean los residuos de cosecha en el valle.
El interés de esta producción también guarda relación con la crianza de otras especies. El 11,9 \% de los productores cuenta solo con cabras, el $20,3 \%$ posee cabras y vacunos, el $15,3 \%$ tiene cabras y ovinos y el 52,5\% tiene cabras, vacunos y ovinos (Fig. 7). En resumen, se puede deducir que la crianza de caprinos en el valle de Cañete, actualmente, registra una tendencia a disminuir y está marcadamente relacionada con la crianza de bovinos lecheros, a causa de factores comerciales y económicos (presencia de acopiadores de leche vacuna en la zona).

La alimentación del ganado caprino tiene como base los residuos de cosecha, llamados rastrojos. Los propietarios carecen de programas formales de alimentación, debido a que en su gran mayoría no recibieron ningún tipo de capacitación y toman como referencia la tradición familiar, la experiencia propia o la observada en otros ganaderos (experiencia empírica) para sus decisiones (Tabla 3). La alimentación se realiza directamente en las zonas donde se encuentran los residuos, debiendo para ello desplazarse con el rebaño diariamente, distancias promedio de dos a cuatro km.

El manejo reproductivo del hato también se lleva a cabo sin ninguna orientación técnica, al encontrarse todos los animales en un solo corral. Según la Fig. 8, el 11,9\% de los propietarios separa a sus animales para un mejor manejo del hato, pero el $88,1 \%$ no lo hace. Este manejo tampoco

Tabla 2. Tipo de crianza

\begin{tabular}{|c|c|c|c|}
\hline \multicolumn{2}{|c|}{ Tipo de crianza } & $\mathbf{N}^{\mathbf{0}}$ de Productores & \% de Productores \\
\hline \multirow{2}{*}{ Intensivo } & Con rastrojo & 3 & $5,1 \%$ \\
\cline { 2 - 4 } & Sin rastrojo & 1 & $1,7 \%$ \\
\hline \multirow{2}{*}{ Rastrojero } & Rastrojero & 50 & $84,7 \%$ \\
\hline \multirow{2}{*}{ Trashumante } & Con rastrojo & 4 & $6,8 \%$ \\
\cline { 2 - 4 } & Sin rastrojo & 1 & $1,7 \%$ \\
\hline
\end{tabular}

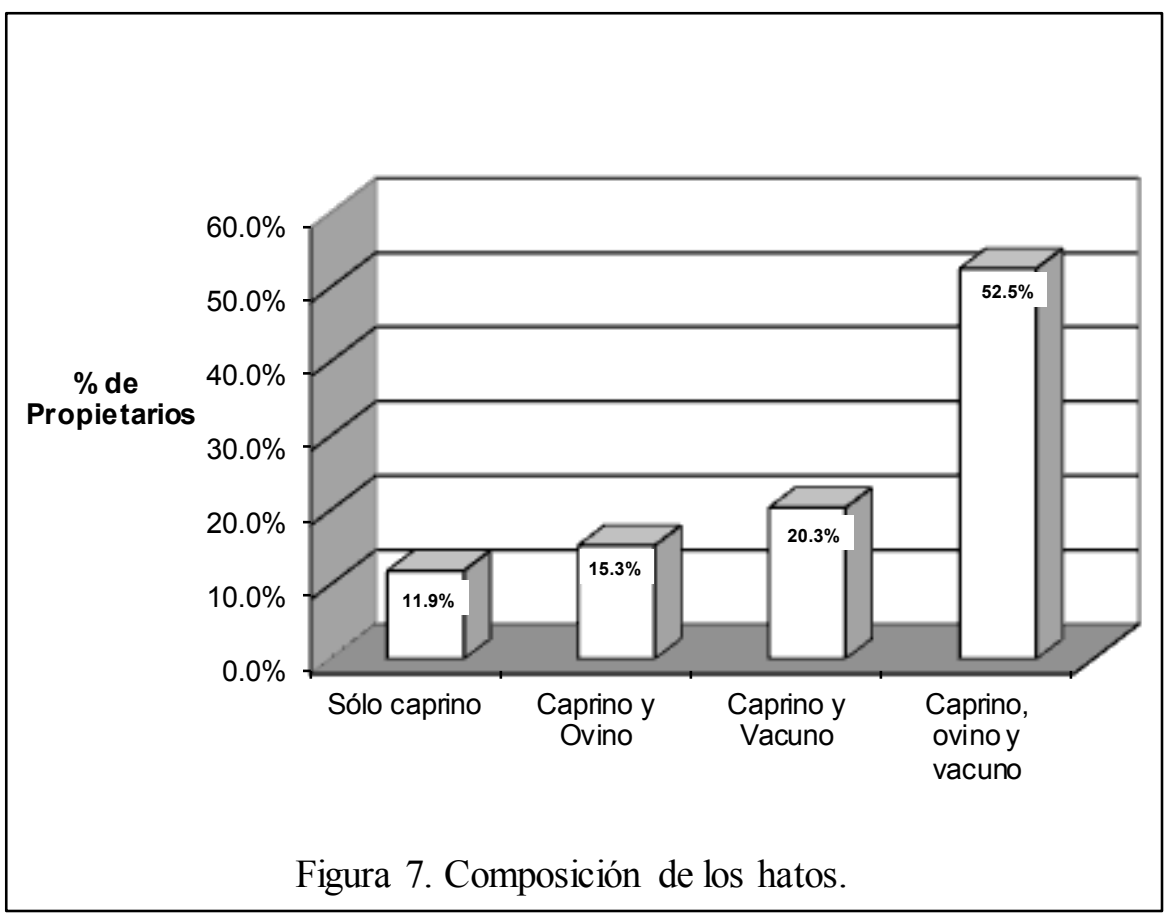


Tabla 3. Criterio de alimentación

\begin{tabular}{|l|c|c|}
\hline \multicolumn{1}{|c|}{ Criterios de Alimentación } & $\mathbf{N}^{\mathbf{0}}$ de Productores & $\begin{array}{c}\text { \% de } \\
\text { Productores }\end{array}$ \\
\hline Tradición familiar & 24 & $40,7 \%$ \\
\hline Observación de otro criador & 1 & $1,7 \%$ \\
\hline Experiencia propia & 8 & $13,6 \%$ \\
\hline Sin criterio & 1 & $1,7 \%$ \\
\hline Tradición + experiencia & 22 & $37,3 \%$ \\
\hline Tradición + sin criterio & 2 & $3,4 \%$ \\
\hline No responde & 1 & $1,7 \%$ \\
\hline \multicolumn{1}{|c|}{ Total } & $\mathbf{5 9}$ & $\mathbf{1 0 0} \%$ \\
\hline
\end{tabular}

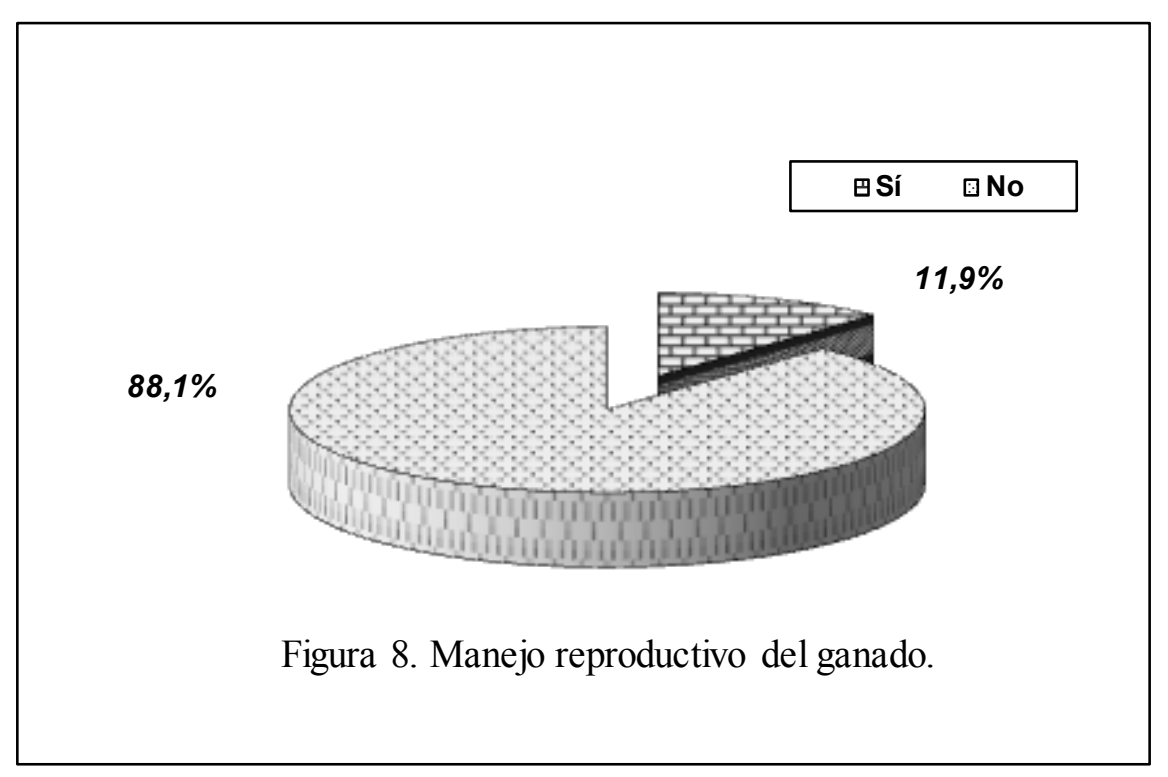

difiere con los reportes encontrados por Arias y Alonso (2002) en Argentina, en donde los productores no utilizan criterios de selección ni control de la reproducción; las hembras y los machos permanecían juntos todo el año. El ordeño se realiza en condiciones extremadamente precarias, debido a que no se cuenta con instalaciones adecuadas. El lugar de ordeño suele ser cualquier ambiente del corral, lo que genera que la leche obtenida adquiera olores y suciedad del ambiente. Todos los cabreros realizan el ordeño a mano, pero al hacerlo no toman las medidas higiénicas necesarias; la limpieza se limita a quitar la tierra de las ubres, lo que hacen incluso sin agua; de otro lado, la recolección se hace en recipientes de aluminio o plástico, todo esto hace que la leche esté expuesta a la tierra y a frecuentes contaminaciones.

La comercialización de los productos de origen caprino se refiere, principalmente y en orden de importancia, a la leche-queso, carne y el estiércol. La comercialización de la leche se hace directamente usando porongos, muchas veces mezclada con la leche de vaca (13,6 \% de los casos). Algunos productores comercializan paralelamente leche y queso $(8,47 \%)$ y la mayoría, $59,3 \%$, comercializa solamente queso. El 18,6 \% comercializa solo cabrito y guano.
Tipos de productores caprinos en el valle de Cañete El procesamiento del integro de la data obtenida en la encuesta no determinó grupos de criadores diferentes entre sí. Sin embargo, seleccionando algunas variables relevantes, que en particular permitían observar diferencias entre criadores, se procedió a caracterizar a los productores de caprinos del valle de Cañete y se halló -por medio del programa SPSS-13- diferentes prototipos de criadores que se dedican a esta actividad productiva, lo cual permite diferenciarlos ya sea por sus caracteres de tipo cuantitativo como de tipo cualitativo.

Las variables consideradas fueron edad, nivel de estudios, áreas de terreno, tamaño de rebaño caprino, número de vacunos, número de ovinos, número de corrales, producción de leche en cantidad y duración de campaña y número de cabras que ordeña.

\section{Análisis multivariante (análisis clúster)}

Como las variables seleccionadas para el análisis tienen diferentes unidades (años, litros, $\mathrm{m}^{2}$, etc.) se tuvieron que estandarizar, considerándose como medida de diferencia entre dos variables la Distancia Euclidiana Cuadrada, que penaliza aquellas variables que presentan mayor 
diferencia; mientras que como criterio de conformación de grupos se siguió el Método de Ward, que es ideal para trabajar con variables estandarizadas. La conformación de grupos se representó en un dendograma, que es una gráfica que establece en el eje de las abscisas a cada uno de los productores de caprinos y en el eje de las ordenadas, las distancias entre cada uno de ellos, distinguiendo los grupos conformados con diferentes colores.

\section{Resultado del análisis multivariante}

El siguiente dendograma representa a los tipos de productores caprinos de Cañete.

Los tipos de productores caprinos encontrados en el valle de Cañete se caracterizan por las variables que se muestran en la Tabla 4.

Las denominaciones asignadas a los tres grupos fueron:

Grupo 1. Productores caprinos de mayor escala productiva (más de 65 cabezas).

Grupo 2. Productores caprinos con mayores áreas de terreno (más de $2.500 \mathrm{~m}^{2}$ ).

Grupo 3. Productores caprinos de mayor edad (más de 60 años).

Lineamientos para el desarrollo de la producción caprina en Cañete

Los lineamientos para el desarrollo de un sistema de producción caprina en el valle del río Cañete, a similitud de otros entornos similares de la costa peruana, deberían formar parte de las futuras agendas de organizaciones, oficinas gubernamentales y asociaciones caprinas, con el objeto de mejorar dichas producciones con un sentido analítico, localmente consensuado y técnicamente consistente.

Para realizar un planteamiento de lineamientos dirigidos al desarrollo del sistema de producción, en este caso la crianza caprina, se debe tener en cuenta ciertos aspectos previos, extraídos de la data y caracterización efectuadas.

\section{Aspectos generales de la caracterización}

- El nivel educativo de una gran mayoría de los productores es solo primario.

- En gran porcentaje, los productores caprinos son veteranos (53,12 años).

- No cuentan con conocimientos suficientes para un buen manejo ni gestión.

- El tipo de explotación en su mayoría es rastrojera.

- El precio de la leche es bajo, con poca promoción y aceptación comercial.

\section{Líneas de acción para el desarrollo caprino de la zona}

- Contar con un Plan local o regional de desarrollo caprino (corto, mediano y largo plazo). En donde se considere los aspectos más críticos de estos productores.

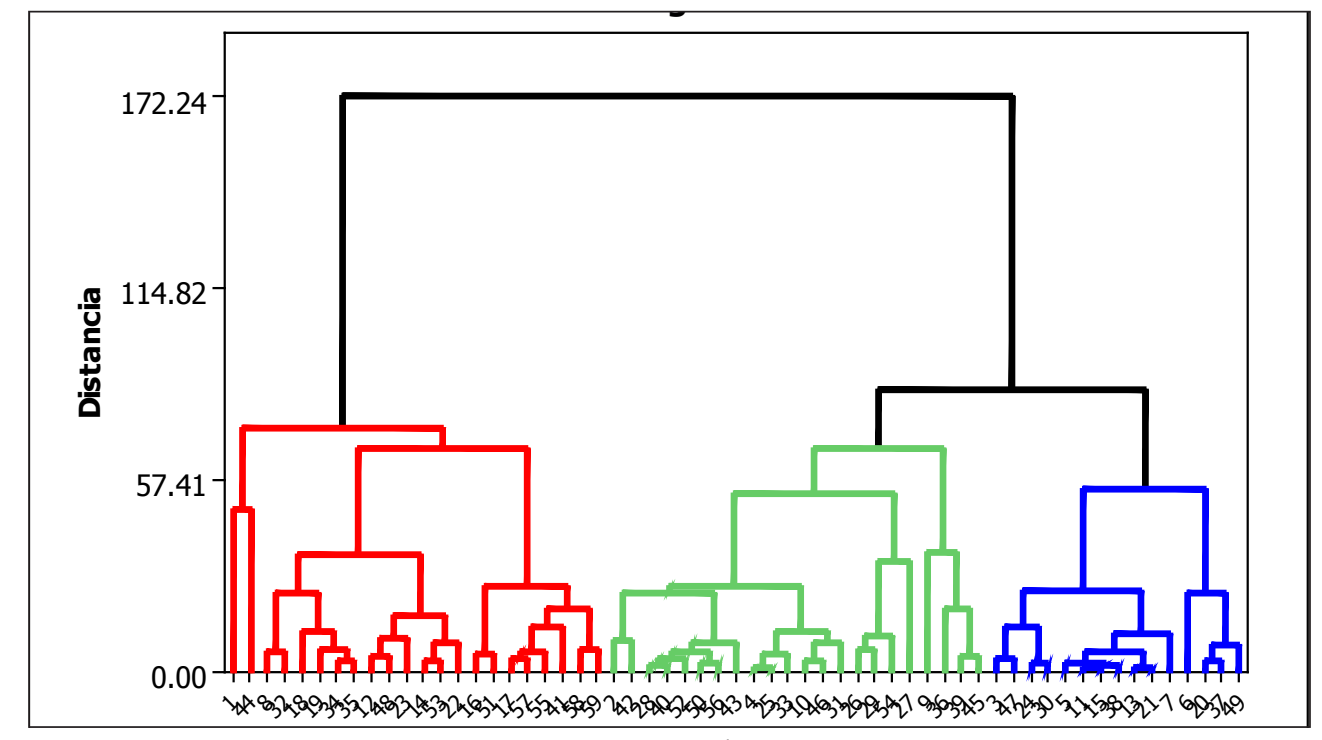

Figura 9. Dendograma

Tabla 4. Valores de las principales variables por tipo de productor

\begin{tabular}{|c|c|c|c|}
\hline Características & Grupo 1 & Grupo 2 & Grupo 3 \\
\hline Edad (años) & 49,1 & 49,8 & 63,8 \\
\hline Estudios (Categ. Prom.) & 2,0 & 2,1 & 2,1 \\
\hline Área $\left(\mathrm{m}^{2}\right)$ & 1227,7 & 2501,8 & 566,0 \\
\hline Rebaño ( $N^{o}$ de animales) & 67,9 & 40,6 & 25,8 \\
\hline Vacunos ( $\mathrm{N}^{\mathrm{o}}$ de animales) & 7,1 & 11,7 & 1,7 \\
\hline Ovinos ( $\mathrm{N}^{\mathrm{o}}$ de animales) & 7,3 & 6,0 & 6,5 \\
\hline Corrales ( $\mathrm{N}^{\mathrm{o}}$ de corrales) & 2,1 & 2,1 & 2,0 \\
\hline Leche (litros / día) & 15,7 & 5,0 & 4,2 \\
\hline Ordeña ( $\mathrm{N}^{\mathrm{o}}$ cabras que ordeña) & 17,1 & 7,0 & 5,3 \\
\hline Tiempo de Prod. (meses) & 4,3 & 3,5 & 4,0 \\
\hline
\end{tabular}


- Realizar capacitaciones de carácter técnico, participativo, con apoyo articulado y planificado de instituciones de prestigio.

- Sensibilizar para concientizar y motivar especialmente a la población joven, a fin de que se dediquen a esta actividad pecuaria, como una ocupación viable y atractiva.

- Promover activamente el asociacionismo, ya que la unión es una vía necesaria para explotar las ventajas comparativas y, sobretodo, lograr ventajas competitivas.

- Mejorar la genética de los animales, así como el manejo productivo conexo.

- Coordinar y promover que la cooperación nacional e internacional apoye coherentemente planes consistentes en el tiempo.

\section{Medidas de planteamiento de acuerdo al tiempo}

a) Medidas de corto plazo (1 a 3 años).- Desarrollar el asociativismo, diseñar y generar un prototipo de criador replicable, descarte de animales improductivos, programa de control de hembras bajo registros y con dos ordeños diarios, construir ambientes adecuados y por categorías, capacitar con buenas prácticas productivas; elaboración de compost a partir del guano.

b) Medidas de mediano plazo (3 a 10 años).- Las explotaciones deberán tender al tipo semi-intensivo o intensivo, ofrecer talleres para elaborar sub productos lácteos de calidad, optimizar buenas prácticas, mejorar la comercialización y programar los nacimientos para que no haya estacionalidad comercial.

c) Medidas de largo plazo (10 años a más).- Consolidar programas de mejora genética por inseminación artificial, fomentar el desarrollo de exposiciones, concursos y alianzas estratégicas.

\section{Conclusiones}

El nivel de instrucción educativa de los criadores de ganado caprino en el valle de Cañete, es esencialmente escolar primario y su edad promedio es de tendencia avanzada. El tipo de explotación en su mayoría es denominada rastrojera $(84,7 \%)$, siendo mayoritariamente asociada con vacunos y ovinos. Los rebaños caprinos son pequeños, teniendo en promedio 46,85 cabezas (rango de 25,8 a 67,9 ). El proceso productivo es en general y definitivamente empírico, acusando como principales problemas la alimentación del hato y la falta de capacitación. La comercialización de los productos caprinos se hace por medio de intermediarios; variando el precio de la leche, queso y carne del cabrito con la época del año, por la oferta estacional existente. El procesamiento del integro de la data obtenida no determinó grupos de criadores diferentes entre sí en el valle de Cañete; sin embargo, la caracterización derivada de variables preestablecidas arrojó los siguientes tipos de productores: (a) Tipo 1: Productores caprinos de mayor escala productiva, (b) Tipo 2: Productores caprinos con mayores áreas de terreno y (c) Tipo 3: Productores caprinos de mayor edad. Finalmente, es necesario desarrollar planes de desarrollo caprino para el corto, mediano y largo plazo, tanto a nivel local como regional, motivando a la población joven a participar mediante la promoción activa del asociacionismo.

\section{Literatura citada}

De Gea, G. 2000. La cabra criolla de las sierras de los Comechingones, Córdoba, Argentina. Córdoba, Argentina: Facultad de Agronomía y Veterinaria, Universidad Nacional de Río Cuarto.

Garcia, A.; Doménech, V.; Frías, J.; Herrera, M.; Peña, F.; Martos, J. y Acero, R. 1999. Caracterización Técnico-Económica de los Sistemas de Producción de Caprino Extensivo en la Provincia de Jaén como base del desarrollo sostenible, pp. 19 - 40. Málaga, España: Junta de Andalucía. Consejería de Agricultura y Pesca.

Gómez, J. 2004. El Método Experimental: Curso Metodología de la investigación Cientifica Avanzada. Lima, Perú.

Mena, Y.; Castel, J.; Pascual, F.; Caravaca, F.; Guzmán, J. y González, P. 2005. Situación actual, evaluación y diagnóstico de los sistemas semiextensivos de producción caprina en Andalucía Centro-Occidental. Junta de Andalucía. Sevilla, España: Ed. Novograf S. L.

Ordoñez, C. 2002. Caracterización de la Comunidad Campesina de Vicos por el tipo de ingreso con fines de implementación de un programa de desarrollo agrícola comunal. (Tesis Mag. Sc.) Universidad Nacional Agraria de La Molina. Lima, Perú.

Venegas, O, R. 2002. Caracterización de los sistemas de producción animal en los caserios ubicados entre los Kilómetros 945-960 de la carretera Panamericana norte - Piura. (Tesis Ing. Zoot.). Facultad de Zootecnia. Universidad Nacional de Piura. Piura, Perú. 